\title{
Multiple cross switching in a two-mode semiconductor laser
}

\author{
Pei-Cheng Ku, Ching-Fuh Lin, ${ }^{\text {a) }}$ and Bor-Lin Lee \\ Graduate Institute of Electro-Optical Engineering and Department of Electrical Engineering, \\ National Taiwan University, Taipei, Taiwan, Republic of China
}

(Received 12 August 1996; accepted for publication 21 October 1996)

\begin{abstract}
A multiple cross switching phenomenon is observed in a two-mode semiconductor laser in an external cavity. Its physical origins are investigated. The changing temperature or injection current causes the variation of the refractive index and the length of the laser diode, leading to the shift of the modulated gain peaks of the anti-reflection-coated laser diode in the external cavity. Consequently, the two modes experience different gains periodically and the strong gain competition then leads to the multiple cross switching. () 1996 American Institute of Physics.
\end{abstract} [S0003-6951(96)02652-6]

Two-mode lasers, in which competition occurs between two modes, could have potential applications in optical communication and optical data processing systems. ${ }^{1,2}$ The two oscillating modes may be two wavelengths, ${ }^{2}$ TE and TM polarization states, ${ }^{3-5}$ or two spatially distinct, but cross coupled laser diodes. ${ }^{6,7}$ In those devices, switching occurs as the intensity of one mode grows significantly to quench the other mode, which then has no chance to regain its intensity. In this letter, we report that in a two-mode semiconductor laser, the two oscillating modes could have multiple switching between each other. The second mode that quenches the first mode can be quenched again and so the first mode regains the intensity. This situation could continue and lead to the multiple cross switching. The physical origins are investigated.

The multiple cross switching is observed from the twomode semiconductor laser set up in the reflected-type grating telescope configuration ${ }^{8}$ that is schematically shown in Fig. 1 . The laser diode used in the setup has a $4 \mu \mathrm{m}$ wide ridgewaveguide structure fabricated on an $\mathrm{AlGaAs} / \mathrm{GaAs}$ quantum-well substrate. The collimated light emitting from the anti-reflection (AR)-coated facet is incident on the grating in the external cavity, so different wavelength components are spatially separate. Double slits are used in the external cavity to force the semiconductor laser to oscillate at two wavelengths. ${ }^{8}$ Output light is obtained from the uncoated facet. The laser diode is temperature-controlled by a TE cooler.

Initially, the two-mode laser is adjusted to oscillate at two modes at a constant biased current and stable cw operation of the two-mode oscillation is observed for hours. However, the two-mode oscillation cannot be maintained for all injection currents without the readjustment of the laser cavity. Figure 2 shows that the intensities of the long- and shortwavelength modes switch to each other several times as the injection current increases beyond the threshold. In the measurement, the alignments of the cavity are not changed. The interesting multiple cross switching is also observed by changing the operation temperature at a constant biased current, as shown in Fig. 3. Several other AR-coated or angled-

a)Electronic mail: cflin@cc.ee.ntu.edu.tw facet laser diodes ${ }^{9}$ have been set up in the same configuration and demonstrated similar phenomena.

Increasing injection current could result in two major effects. One is the band filling, which will cause the oscillation preferred at the short-wavelength mode. From our measurements in pulsed operation, the injection current could cause the wavelength blue-shifted by $\sim 0.294 \AA / \mathrm{mA}$. The other effect is the temperature rising that leads to the band gap shrinkage, so oscillation is preferred at the longwavelength mode. Hence, with the injection current fixed, increasing temperature results in a red-shifted wavelength at a rate of $\sim 2.68 \AA /{ }^{\circ} \mathrm{C}$ for our devices. In cw operation, the injection current also heats up the device and the increase of temperature is measured to be $0.33{ }^{\circ} \mathrm{C} / \mathrm{mA}$. It appears that the multiple cross switching in the $\mathrm{cw}$ operation might result from the interplay between the above two effects. However, multiple cross switching between the long- and shortwavelength modes also occurs with only the temperature variation, as shown in Fig. 3. Therefore, the above two effects cannot fully explain this phenomenon and further investigation is required.

In an external-cavity configuration, the residual reflectivity at the AR-coated facet of a laser diode could cause the coherent interference effects in the compound cavity. ${ }^{10}$ As a result, the gain profile is modulated by the Fabry-Perot resonance of the laser diode. The modulation depth of the gain

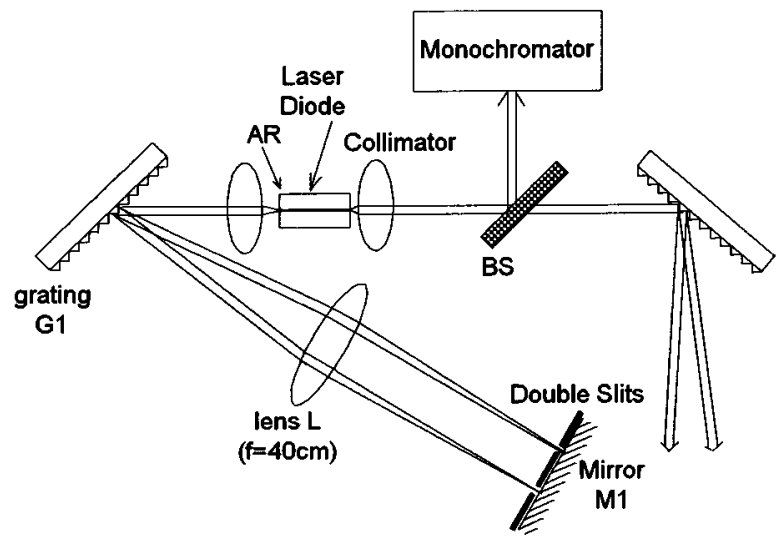

FIG. 1. A schematic of the two-mode semiconductor laser for the experiment of multiple cross switching. 


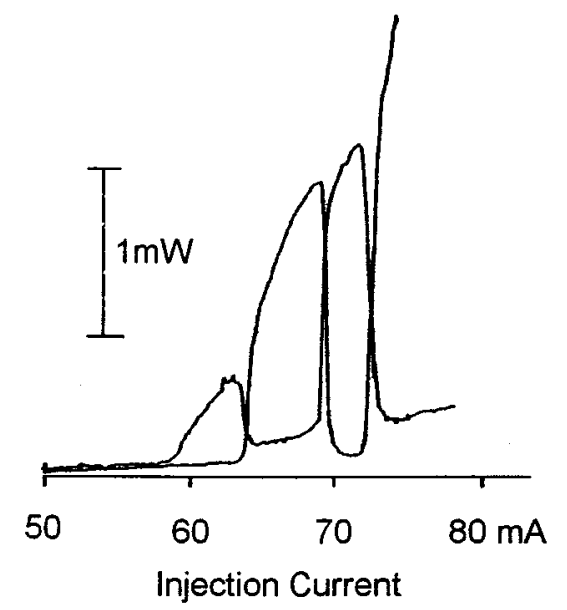

FIG. 2. Multiple cross switching between the long- and short-wavelength modes with increasing injection current. The mode spacing is $8 \mathrm{~nm}$.

profile in such a compound cavity can be estimated according to the following formula ${ }^{10,11}$

$$
g_{\max }-g_{\min }=\frac{2 r_{2}}{r_{1}} \frac{1-C^{2} r_{3}^{2}}{C^{2} r_{3}^{2}-r_{2}^{2}},
$$

where $r_{1}, r_{2}$, and $r_{3}$ are the field reflectivities of the laserdiode cleaved facet, the AR-coated facet, and the external mirror, respectively; $C$ is the coupling coefficient (with the collimator and the grating efficiency taken into account). This modulation depth is over $1 \%$ if the AR-coated facet of the laser diode in the external cavity has a power reflectivity larger than $1 \times 10^{-4}$. In addition, the modulation peaks of the gain profile correspond to the Fabry-Perot modes of the laser diode. Their frequencies are given by

$$
\nu_{m}=\frac{m c}{2 n L},
$$

where $n$ is the effective index and $L$ is the length of the laser diode; $c$ is the speed of light; $m$ is a positive integer that approximately equals 2500 for the $850 \mathrm{~nm} \mathrm{GaAs} / \mathrm{AlGaAs}$ laser diode used in the experiment.

Equation (2) shows that those peak positions depend on the values of $n$ and $L$, which however vary with the temperature. ${ }^{12}$ In addition, the injection current could cause

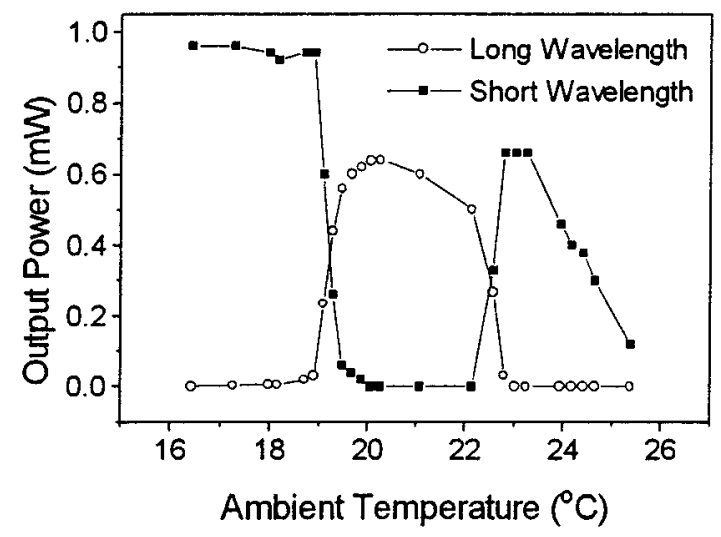

FIG. 3. Multiple cross switching between the long- and short-wavelength modes with temperature variation. The mode spacing is $6 \mathrm{~nm}$. (a)
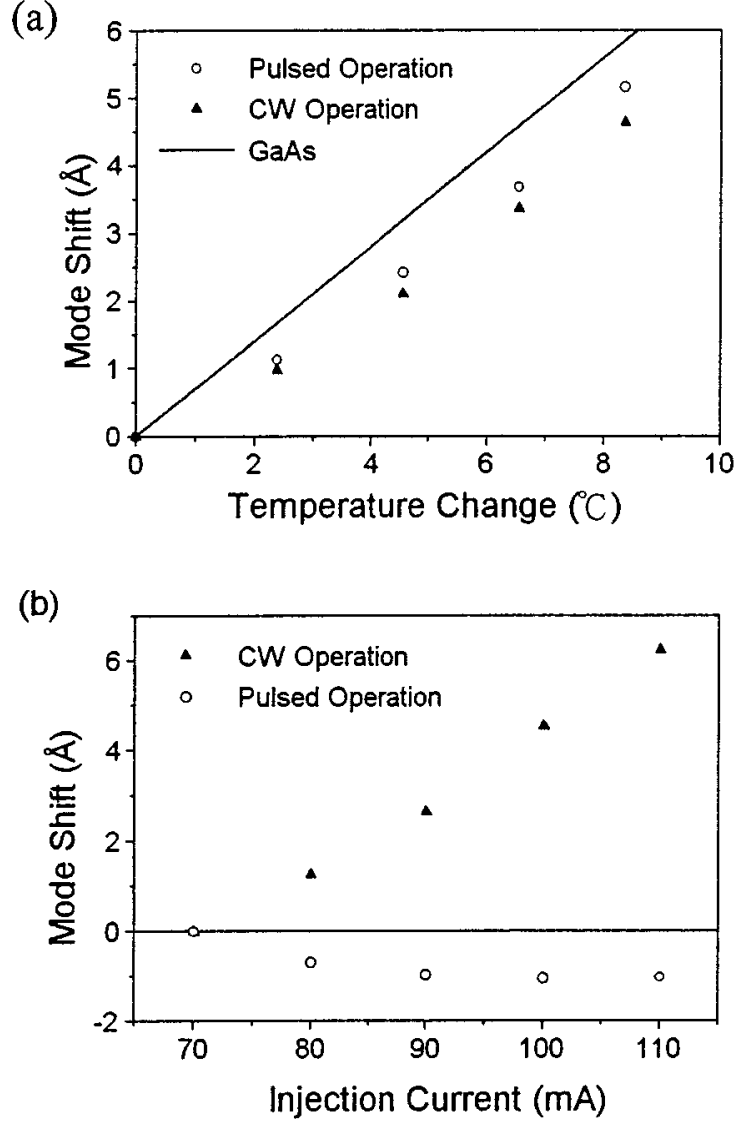

FIG. 4. (a) The dependence of the Fabry-Perot mode shift with the increasing ambient temperature. The solid line is calculated from the data of GaAs. (b) The dependence of the Fabry-Perot mode shift with the increasing injection current.

the refractive-index change due to the carrier injection. In the $\mathrm{cw}$ operation, the injection current also induces temperature increase, which, in turn, changes the device length. Figures 4(a) and 4(b) show the measured mode shift versus the temperature change and the injection current, respectively. Theoretical calculation of mode shift versus temperature change based on the GaAs data of thermal expansion coefficient and refractive-index temperature coefficient ${ }^{12}$ is also plotted in Fig. 4(a). The measurements are very close to the theoretical calculation. The slight discrepancy is possibly because the quantum well structure instead of the pure GaAs material is used for the fabrication of the devices. Due to the large value of $m$ in Eq. (2), the mode shift is significant even though the change of temperature or injection current is small.

Now the multiple cross switching because of the injection current and the temperature change can be realized. In the experiment, the positions of the two slits are fixed, while the gains corresponding to the slit positions vary with the injection current and the temperature. Figure 5 illustrates such gain modulation under different conditions. The modulation period corresponds to the diode-chip mode spacing instead of the external-cavity mode spacing. As shown in Fig. 5, at temperature (or injection current) $a$, slit 1 selects a larger gain than slit 2. At temperature (or injection current) $b$, slits 1 and 2 select equal gains, while at temperature (or injection current) $c$, slit 1 selects a smaller gain than slit 2 . However, further increasing the temperature (or injection 


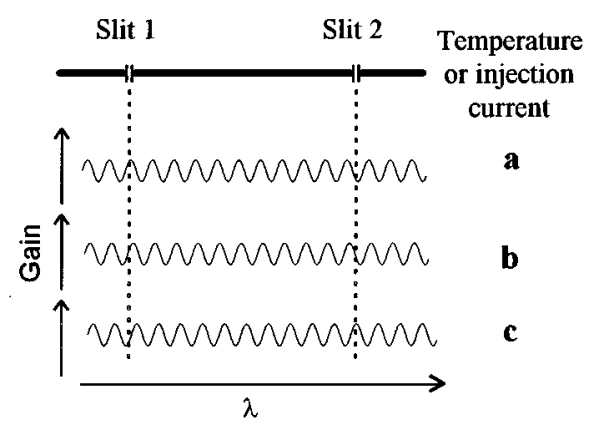

FIG. 5. Illustration of the gain difference between modes 1 and 2 at different temperatures or injection currents.

current) could cause slit 1 to select a larger gain again due to the periodic variation of the gain peaks. The situation continues and so multiple switching between mode 1 and 2 is observed.

It might be questionable if the gain difference between mode 1 and mode 2 due to the gain modulation in the compound cavity is sufficient for the switching. The condition for the optical switching in such a two-mode semiconductor laser can be analyzed using the two-mode rate equations: ${ }^{13}$

$$
\begin{aligned}
& \frac{d I_{1}}{d t}=\left(g_{1}-l_{1}-g_{1} S_{1} I_{1}-g_{1} C_{12} I_{2}\right) I_{1}, \\
& \frac{d I_{2}}{d t}=\left(g_{2}-l_{2}-g_{2} S_{2} I_{2}-g_{2} C_{21} I_{1}\right) I_{2},
\end{aligned}
$$

where the $S \mathrm{~s}$ and $C$ s are self-saturation and cross-saturation coefficients, respectively; $g \mathrm{~s}$ are the unsaturated gain and $l$ 's are the cavity loss of each mode. It can be shown that the simultaneous oscillation of modes 1 and 2 only occurs under the following condition:

$$
\frac{C_{21}}{S_{1}}<\left(1-\frac{l_{2}}{g_{2}}\right) /\left(1-\frac{l_{1}}{g_{1}}\right)<\frac{S_{2}}{C_{12}} .
$$

Beyond this range, the stable oscillation will be either at mode 1 or 2 , but not both. Therefore, switching between modes 1 and 2 should occur if gain or loss varies beyond the range given by Eq. (4). In addition, this range becomes very small as the ratios, $C_{21} / S_{1}$ and $C_{12} / S_{2}$, approach unity. This could be the situation for a two-mode laser with a very strong gain competition. Therefore, switching between these two modes could be achieved via only a very small amount of loss or gain modulation. Experimentally it has been found that about $1 \%$ of additional loss inserted in one mode is sufficient to cause the switching in such a two-mode semiconductor laser. For the setup shown in Fig. 1, the gain modulation depth is estimated to be over $1 \%$ for the ARcoated facet with a power reflectivity larger than $1 \times 10^{-4}$. Therefore, the switching could happen due to the gain modulation caused by the residual reflectivity of the AR-coated facet in the external-cavity semiconductor lasers.

In conclusion, multiple cross switching is observed in two-mode semiconductor lasers. The two oscillating modes switch to each other many times as the injection current increases or the operation temperature varies. The physical origins are investigated. The change of temperature or injection current results in a variation of the refractive index and the length of the laser diode, leading to the mode shift. Then, the modulated gain peaks of the AR-coated laser diode in the external cavity also shift. As a result, the two modes experience different gains periodically and so the multiple cross switching occurs because of the strong gain competition between the two modes.

This work is supported in part by National Science Council, Taipei, Taiwan, Republic of China, under Contract No. NSC85-2112-M-002-018.

${ }^{1}$ K.-Y. Liou, C. A. Burrus, U. Koren, and T. L. Koch, Appl. Phys. Lett. 51, 1777 (1987)

${ }^{2}$ H. Shoji, Y. Arakawa, and Y. Fujii, IEEE Photonics Technol. Lett. 2, 109 (1990)

${ }^{3}$ Y. C. Chen and J. M. Liu, Opt. Quantum Electron. 19, S93 (1987).

${ }^{4}$ H. Tanaka, J. Shimada, and Y. Suzuki, Appl. Phys. Lett. 64, 158 (1994).

${ }^{5}$ A. Klehr, R. Muller, M. Voss, and A. Barwolff, Appl. Phys. Lett. 64, 830 (1994).

${ }^{6}$ H. Uenohara, Y. Kawamura, H. Iwamura, K. Nonaka, H. Tsuda, and T. Kurokawa, Electron. Lett. 28, 1973 (1992).

${ }^{7}$ J. E. Johnson and C. L. Tang, Appl. Phys. Lett. 63, 3273 (1993).

${ }^{8}$ Ching-Fuh Lin, 1995 Semiconductor lasers: Advanced Devices and Applications, Keystone, Colorado, 1995, paper TuE8.

${ }^{9}$ C. F. Lin, IEEE Photonics Technol. Lett. 4, 127 (1992).

${ }^{10}$ A. Olsson and C. L. Tang, IEEE J. Quantum Electron. 17, 1320 (1981).

${ }^{11}$ Ching-Fuh Lin, Ph.D. dissertation, Cornell University, 1993.

${ }^{12}$ Properties of Aluminum Gallium Arsenide, edited by S. Adachi (INSPEC, London, 1993).

${ }^{13}$ A. E. Siegman, Lasers (University Science Books, 1986). 
Applied Physics Letters is copyrighted by the American Institute of Physics (AIP). Redistribution of journal material is subject to the AIP online journal license and/or AIP copyright. For more information, see http://ojps.aip.org/aplo/aplcr.jsp

Copyright of Applied Physics Letters is the property of American Institute of Physics and its content may not be copied or emailed to multiple sites or posted to a listserv without the copyright holder's express written permission. However, users may print, download, or email articles for individual use. 